GUEST EDITORIAL

\title{
Dyslipidaemia in South Africa
}

Cardiovascular disease (CVD) is the leading cause of death worldwide, with an estimated 15 million people dying from ischaemic heart disease and stroke in 2015, which represents $26 \%$ of all deaths that year. ${ }^{[1]}$ CVD disproportionately impacts low- and middle-income countries, where $\sim 80 \%$ of the global CVD mortality occurs. ${ }^{[1]}$ Dyslipidaemia is an important risk factor and a primary driver for CVD, and is defined by the presence of suboptimal serum lipid levels that portend heightened risk of cardiovascular events. There is a paucity of literature on the epidemiology and management of dyslipidaemia in South Africa (SA).

The Health and Aging in Africa: A Longitudinal Study of an INDEPTH Community in South Africa (HAALSI) study ${ }^{[2]}$ was designed to assess the awareness, treatment and control of dyslipidaemia in rural SA, and reported that $67 \%$ of rural South Africans met criteria for dyslipidaemia; only $30 \%$ of them were aware of their condition and only $0.7 \%$ were receiving treatment. In the HAALSI study, ${ }^{[2]}$ being overweight was predictive of dyslipidaemia and dyslipidaemia awareness. These data are particularly concerning when considered against the background of rising levels of obesity, especially among rural women, as well as the increasing vulnerability of rural SA populations, who have limited access to both information and optimal medical therapy.

In the Dyslipidemia International Study (DYSIS), ${ }^{[3]}$ a crosssectional, observational study of lipid control in patients receiving statin therapy, the SA participants $>45$ years old and who had been treated with a stable dose of a statin for 3 years, had lipid goal attainment investigated. DYSIS reported that $50 \%$ of the SA patients did not achieve target low-density lipoprotein cholesterol (LDL-C) levels and $74 \%$ of these patients were at very high risk of CVD. Furthermore, $34 \%$ had low high-density lipoprotein cholesterol (HDL-C) levels and 45\% had elevated triglyceride (TG) levels despite statin therapy ${ }^{[3]}$ Persisting lipid abnormalities in the context of suboptimal therapy place patients at ongoing risk of CVD, particularly many SA public sector patients who receive very low doses of statins, with lipid profiles not followed up after initiation of therapy.

A retrospective study performed on black SA patients treated at the Dr George Mukhari Hospital, Gauteng, found a significant burden and high prevalence of dyslipidaemia in black adults in whom a monogenic disorder should have been considered. ${ }^{[4]}$ Severe hypercholesterolaemia (TG $>7 \mathrm{mmol} / \mathrm{L}$ ) was seen in $5 \%$ and extreme hypercholesterolaemia (TG $>12 \mathrm{mmol} / \mathrm{L}$ ) in $0.5 \%$ of patients. Elevated TG levels were noted in $9 \%$ of patients. The authors concluded that the extent and severity of the dyslipidaemia justified a special clinic and laboratory to ensure accurate diagnosis, with effective intervention for patients and their families. ${ }^{[4]}$ Indeed, there are very few dedicated, specialist lipid clinics in the public and private sectors in SA.

The association between dyslipidaemia and anthropometric indicators in black and white adolescents (aged 12 - 16 years) residing in the North West Province of SA has been investigated in the Physical Activity and Health Longitudinal (PAHL) study. ${ }^{[5]}$ This study found that waist circumference correlated negatively with HDL-C in both black and white children, and in white children it also correlated positively with LDL-C, total cholesterol (TC)/HDL-C and LDL-C/ HDL-C ratios. In white children, there were greater associations between dyslipidaemia and anthropometric indicators, suggesting that there might be differences in lipid metabolism or susceptibility to risk factors at this young age. ${ }^{[5]}$ These biological risk factors are further complicated by lack of physical activity and unhealthy diets among young South Africans.

In the SA population, diabetes is an important driver of dyslipidaemia. A publication on lipid levels in black South Africans with type 2 diabetes confirmed this association. ${ }^{[6]}$ Diabetes often coexists with other cardiovascular risk factors, such as hypertension, obesity, smoking, gout and hypercoagulability, all of which significantly increase the risk of CVD. SA has the largest population of people living with HIV worldwide, who receive antiretroviral therapy (ART), and also a significant number of treated HIV-infected persons with dyslipidaemia. In a recent publication, Dave et al. ${ }^{[7]}$ reported the prevalence of dyslipidaemia to be $90 \%$ in ART-naive and $85 \%$ in treated HIV-infected South Africans.

The SA Dyslipidaemia Guideline Consensus Statement, ${ }^{[8]}$ based on the European Society of Cardiology and European Atherosclerosis Society guidelines, for the management of dyslipidaemia, was published in 2012, under the auspices of the SA Heart Association and the Lipid and Atherosclerosis Society of Southern Africa. The objective of these simplified guidelines was to promote current best management of dyslipidaemia in SA.

In this issue of $\mathrm{CME}$, Blom ${ }^{[9]}$ provides an excellent review on an approach to the patient with elevated TGs. Mild-to-moderate hypertriglyceridaemia (TG $>1.7-10 \mathrm{mmol} / \mathrm{L}$ ) is an independent cardiovascular risk factor, while severe hypertriglyceridaemia (TG $>10.0 \mathrm{mmol} / \mathrm{L}$ ) can cause acute pancreatitis. Hypertriglyceridaemia is mostly polygenic. Secondary factors, such as uncontrolled diabetes, hypothyroidism, alcohol, renal disease and medications, are important in precipitating or exacerbating hypertriglyceridaemia. Patients with hypertriglyceridaemia require cardiovascular risk assessment and are in the first instance treated with statins if their cardiovascular risk is elevated. Control of secondary factors and lifestyle modifications are important in managing residual hypertriglyceridaemia in statintreated patients. Severe hypertriglyceridaemia is managed by removing or controlling secondary factors, by following a very low-fat diet and by prescribing a fibrate.

Brozin and Raal ${ }^{[10]}$ provide a state-of-the-art review on novel approaches to lipid-lowering therapy, focusing on new drugs and their mechanisms of action. Statins (with or without ezetimibe) and fibrates are the current first-line therapy in the management of dyslipidaemia. Novel agents include the proprotein convertase subtilisin/kexin type 9 (PCSK9) inhibitors (that have shown to lead to a reduction in CVD morbidity), mipomersen, cholesterol ester transfer protein (CETP) inhibitors and bempedoic acid. Drugs such as pemfibrate, angiopoietin-like protein 3 (ANGPTL3) inhibitors, apolipoprotein C3 (apo C3) inhibitors and diacylglycerol acyltransferase-1 (DGAT 1) inhibitors have shown promising results in the management of hypertriglyceridaemia.

Finally, Rusch et al. ${ }^{[11]}$ provide an up-to-date, pragmatic and evidence-based approach to the laboratory investigations in lipidology. The conventional lipid profile, comprising fasting TG, TC, HDL-C and LDL-C, suffices for screening persons at risk for atherosclerosis. Non-fasting samples are gaining in popularity. In severe dyslipoproteinaemia, special investigations establish the cause of the disease and may allow selection of better treatment. In such cases, not only apoprotein concentrations, but also enzyme or cell 
function, as well as genetic investigations, are relevant; important genetic disorders to recognise are familial hypercholesterolaemia, dysbetalipoproteinaemia and chylomicronaemia.

It is my hope that the articles in this CME issue will improve the investigation and management of dyslipidaemia in SA, thereby reducing the risk of CVD in our population.

Funding. This manuscript is not funded. Prof. N Ntusi gratefully acknowledges support from the National Research Foundation and the Medical Research Council of South Africa, as well as the Harry Crossley Foundation.

\section{Conflicts of interest. None.}

\section{Ntobeko Ntusi}

Division of Cardiology, Department of Medicine, Faculty of Health Sciences, University of Cape Town and Groote Schuur Hospital; Cape Universities Body Imaging Centre, Faculty of Health Sciences, University of Cape Town; and Hatter Institute of Cardiovascular Research in Africa, Department of Medicine, Faculty of Health Sciences, University of Cape Town, South Africa ntobeko.ntusi@uct.ac.za

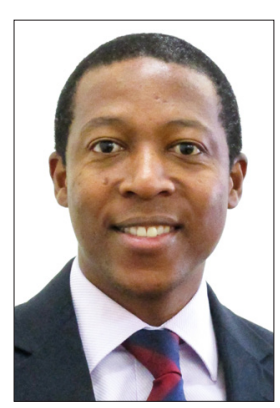

1. World Health Organization. Global Status Report on Noncommunicable Diseases 2015. Geneva: WHO, 2016.

2. Reiger S, Jardim TV, Abrahams-Gessel S, et al. Awareness, treatment, and control of dyslipidemia in rural South Africa: The HAALSI (Health and Aging in Africa: A Longitudinal Study of an INDEPTH Community in South Africa) study. PLOS ONE 2017;12(10):e0187347. https://doi.org/10.1371/ journal.pone. 0187347

3. Raal FJ, Blom DJ, Naidoo S, Bramlage P, Brudi P. Prevalence of dyslipidaemia in statin-treated patients in South Africa: Results of the Dyslipidaemia International Study (DYSIS). Cardiovasc J Afr 2013;24(8):330-338. https://doi.org/10.5830/CVJA-2013-071

4. Khine AA, Marais DA. High prevalence of primary dyslipidaemia in black South African patients at a tertiary hospital in northern Gauteng, South Africa. S Afr Med J 2016;106(7):724-729. https://doi. org/10.7196/SAMJ.2016.v106i7.10337

5. Mamabolo RL, Sparks M, Moss SJ, Monyeki MA. The association between dyslipidemia and anthropometric indicators in black and white adolescents residing in Tlokwe Municipality, NorthWest Province, South Africa: The PAHL study. Afr Health Sci 2014;14(4):929-938. https://doi. org/10.4314/ahs.v14i4.23

6. Joffe BI, Distiller LA, Kalk WJ. Lipid levels in black South Africans with type 2 diabetes. Diabet Care 2004;27(7):1839-1840.

7. Dave JA, Levitt NS, Ross IL, Lacerda M, Maartens G, Blom D. Antiretroviral therapy increases the prevalence of dyslipidaemia in South African HIV-infected patients. PLOS ONE 2016;11(3):e0151911. https://doi.org/10.1371/journal.pone.0151911

8. Klug E; South African Heart Association (S A Heart); Lipid and Atherosclerosis Society of Southern Africa (LASSA). South African dyslipidaemia guideline consensus statement. S Afr Med J 2012;102(3):178-187.

9. Blom DJ. Elevated triglycerides: A matter of the heart and pancreas. S Afr Med J 2018;108(4):258-261. https://doi.org/10.7196/SAMJ.2018.v108i4.13235

10. Brozin D, Raal FJ. Novel approaches to lipid-lowering therapy. S Afr Med J 2018;108(4):262-265. https://doi.org/10.7196/SAMJ.2018.v108i4.13234

11. Rusch JA, Hudson CL, Marais AD. Laboratory investigations in lipidology. S Afr Med J 2018;108(4):266-270. https://doi.org/10.7196/SAMJ.2018.v108i4.13233

S Afr Med J 2018;108(4):256-257. DOI:10.7196/SAMJ.2018.v108i4.13265 